\title{
Small-angle neutron scattering study of sodium cholate and sodium deoxycholate interacting micelles in aqueous medium
}

\author{
J SANTHANALAKSHMI* $^{1}$, G SHANTHA LAKSHMI ${ }^{1}$, V K ASWAL ${ }^{2}$ and \\ P S GOYAL ${ }^{2}$ \\ ${ }^{1}$ Department of Physical Chemistry, University of Madras, Guindy Campus, \\ Chennai 600 025, India \\ ${ }^{2}$ Condensed Matter Physics Division, Bhabha Atomic Research Division, \\ Trombay, Mumbai 400 085, India \\ e-mail: jaysune@eth.net
}

MS received 21 July 2000; revised 23 November 2000

\begin{abstract}
Small angle neutron scattering (SANS) measurements of $\mathrm{D}_{2} \mathrm{O}$ solutions $(0.1 \mathrm{M})$ of sodium cholate $(\mathrm{NaC})$ and sodium deoxycholate $(\mathrm{NaDC})$ were carried out at $T=298 \mathrm{~K}$. Under compositions very much above the critical micelle concentration (CMC), the bile salt micelle size growths were monitored by adopting Hayter-Penfold type analysis of the scattering data. $\mathrm{NaC}$ and $\mathrm{NaDC}$ solutions show presence of correlation peaks at $Q=0 \cdot 12$ and $0 \cdot 1 \AA^{-1}$ respectively. Monodisperse ellipsoids of the micelles produce best fits. For $\mathrm{NaC}$ and $\mathrm{NaDC}$ systems, aggregation number $(9 \cdot 0$, $16 \cdot 0)$, fraction of the free counterions per micelle $(0 \cdot 79,0 \cdot 62)$, semi-minor $(8 \cdot 0 \AA)$ and semi-major axes $(18.4,31.7 \AA)$ values for the micelles were deduced. Extent of micellar growth was studied using ESR correlation time measurements on a suitable probe incorporating $\mathrm{NaC}$ and $\mathrm{NaDC}$ micelles. The growth parameter (axial ratio) values were found to be 2.3 and 4.0 for $\mathrm{NaC}$ and $\mathrm{NaDC}$ systems respectively. The values agree with those of SANS.
\end{abstract}

Keywords. Biosurfactants; sodium deoxycholate micelles; bile salt micelles; SANS study; ESR correlation times.

\section{Introduction}

The role of bile salts (sodium cholate, $\mathrm{NaC}$; sodium deoxycholate, $\mathrm{NaDC}$ ) as physiological surfactants is based on their micelle-forming properties ${ }^{1-3}$. These represent the class of potential biosurfactants which play active roles as cholesterol and lipid solubilizers, emulsifiers and dispersion agents in cosmetics, medicines and chemicals ${ }^{4-6}$. Micellar properties of $\mathrm{NaC}$ and $\mathrm{NaDC}$ at nearly their respective critical micelle concentration (CMC) values have been reported in the literature based on different experimental techniques ${ }^{7-10}$. Also, $\mathrm{NaC}, \mathrm{NaDC}$ and other bile salts exhibit concentrationdependent microstructures ${ }^{11}$. The vital bioactivities of bile salts are found at concentrations very much above $\mathrm{CMC}$ and are due to their micellar properties. Near $\mathrm{CMC}$ values, primary micelles with low aggregation numbers are detected, which at higher concentrations produce secondary or rod-like super-grown micelles ${ }^{12,13}$. In the $\mathrm{NaC}$ system, the $\mathrm{CMC}$ value for the primary micellization occurs around 0.013-0.018 M

*For correspondence 
corresponding to the primary aggregates consisting of dimer units. At higher concentrations of $\mathrm{NaC}$, around $0.05 \mathrm{M}$, secondary micellization is detected corresponding to enlarged dimensions of primary micelles ${ }^{14,15}$. Similarly for the NaDC system, around $0.01 \mathrm{M}$ and $0.05 \mathrm{M}$ primary and secondary micellizations are detected in presence of $p \mathrm{H}$ buffers. With further increase in the concentration of bile salts, a third micellization may set in, which may be super-grown secondary micelles with extended dimensions in size. In all, bile salt aggregation behaviour is strongly concentration-dependent and units of dimer are stacked through successive hydrophobic and $\mathrm{H}$-bonds. Based on the experimental technique being adopted, accuracy in the detection of various stages of stepwise aggregation may be rendered possible. Surface tension studies on bile salt system under $p \mathrm{H}$ control prove to be of such kind. In such cases, exact estimations of the aggregation number, interaction potentials of micelles and the nature of the extended size growths in micelles are still ambiguous. Earlier reports on SANS of bile salts consist of radii and rod axis lengths, with $p \mathrm{H}$ and ionic strength effects ${ }^{16-19}$.

The present work is devoted to adopting small angle neutron scattering (SANS) which is a powerful tool to investigate self-association patterns, for the study of secondary micelles of $\mathrm{NaC}$ and $\mathrm{NaDC}$ at $0 \cdot 1 \mathrm{M}$ concentrations in the absence of any additives. Scattering patterns are subjected to Hayter-Penfold analysis and using correlation peak positions, determination of many physicochemical parameters of the micelles such as the equivalent spherical radius $\left[R=\left(a^{2} b\right)^{1 / 3}\right]$, semi-minor and semi-major axes $(a, b)$, number density of micelles $\left(N_{m}\right)$, surfactant aggregation number $\left(N_{B S}\right)$, fraction of the free counterions per micelle $\left(\alpha_{B S}\right)$, micelle concentration [mic], Debye screening length $\left(\kappa^{-1}\right)$ and the mean intermicellar interaction potentials $(U(r) / U(T))$ from SANS are carried out for $\mathrm{NaC}$ and $\mathrm{NaDC}$ micelles at $298 \mathrm{~K}$. In aqueous systems, the hydrodynamic equivalent spherical radii of the ionic secondary micelles vary sensitively with environmental conditions like $p \mathrm{H}$, salt, temperature etc., just as they do for primary micelles ${ }^{20,21}$. Hence evaluation of microstructures of secondary micelles is essential. The evaluation of $R_{h}$ of the bile salt micelles under compositions very much above CMC may be carried out using an ESR spin label technique ${ }^{22}$. In the present work, an anisotropy sensitive spin label such as 3-(2,2'-dimethyl-N-oxyl-oxazolidinyl)-5 $\alpha$-cholestane (CSL) $\left(1 \times 10^{-4} \mathrm{M}\right)$ incorporated $\mathrm{NaC}$ and $\mathrm{NaDC}(0.1 \mathrm{M})$ micelle solutions are subjected to ESR measurements ${ }^{23,24}$. The analysis of the spectral line shapes and line widths can be performed in terms of rotational correlation time $\left(\tau_{R}\right)$, which when applied with StokesEinstein equation, the effective hydrodynamic radius value of the micelle may be determined. Also the semi-major and semi-minor axes values of the ellipsoidal micelles are computed. ESR and SANS results of the bile salt micelles are compared and discussed.

\section{Experimental}

\subsection{Materials}

$\mathrm{NaC}$ and $\mathrm{NaDC}$ samples from Sigma Chemical Co. (USA) were used as such. Sigma $\mathrm{D}_{2} \mathrm{O}$ was used instead of water. Quantitatively weighed amounts of the bile salts and $\mathrm{D}_{2} \mathrm{O}$ were mixed and sonicated to prepare isotropically homogenous solutions. The spin probe CSL was also purchased from Sigma and was used without further purification. ESR samples were prepared by dissolving the spin probe in ethanol which was evaporated in a sample tube using nitrogen purging during which the bile salt solution was added quantitatively under constant stirring and in a sealed condition. 


\subsection{SANS measurements}

SANS measurements were carried out on the spectrometer fitted with a one-dimensional position sensitive detector installed at the Dhruva Reactor, Trombay, Bhabha Atomic Research Centre, Mumbai. The experimental procedure adopted was according to the User's manual $^{25}$. The liquid samples were taken in a quartz cell of path length $1 \mathrm{~cm}$. A $\mathrm{BeO}$ filtered neutron beam of mean wavelength $\lambda=5.2 \AA$ was used. The wavevector transfer $Q$ range accessible from the instrument was 0.02 to $0.08 \AA^{-1}$ where $Q=4 \pi \sin \theta / \lambda$ and $2 \theta$, the scattered angle. The data were collected for the fast neutron background up to $Q=0 \cdot 3 \AA^{-1}$ and the transmission factor was normalised to the absolute cross-section units. Based on the conclusions of Berr ${ }^{26}$ the micellar properties in $\mathrm{D}_{2} \mathrm{O}$ were assumed to be unaltered from that of $\mathrm{H}_{2} \mathrm{O}$ solutions, within the limits of experimental error.

The measured SANS distribution was analysed using the method of Hayter-Penfold for an assembly of monodisperse uniform ellipsoidal micelles. The coherent differential cross-section $(\mathrm{d} \Sigma / \mathrm{d} \Omega)$ can be expressed $\operatorname{as}^{27}$,

$$
\mathrm{d} \Sigma / \mathrm{d} \Omega=N_{m} V_{m}^{2}\left(\rho_{m}-\rho_{s}\right)^{2}\left[\left\langle F^{2}(Q)\right\rangle+\langle F(Q)\rangle^{2}\{S(Q)-1\}\right],
$$

where $N_{m}$ is the number density of the micelles, $\rho_{m}$ and $\rho_{s}$ are the scattering length densities of micelle and solvent, $V_{m}$ is the micelle volume, $F(Q)$ is the form factor defined as $\left(1 / V_{m}\right) \int \exp (i Q \Sigma) \mathrm{d} \Sigma$ that describes the angular distribution of scattering to the morphology of the micelle with respect to $Q$ and $Q$ being $4 \pi \sin \theta / \lambda$ respectively.

The structure factor $S(Q)$ represents interparticle interferences and has been calculated approximating the ellipsoid to an equivalent sphere of radius $R=\left(a^{2} b\right)^{1 / 3}$, where $R$ is the effective radius of the micelle. The concentration of micelles [mic] $\mathrm{mol} \mathrm{dm}{ }^{-3}$ is known from $N_{B S}, C_{s}$ and $\mathrm{CMC}$ as equal to $\left(C_{s}-\mathrm{CMC}\right) / N_{B S}$. The number density of micelle in solution $N_{m}=N_{a v}[\mathrm{mic}] / 1000\left(\mathrm{ml}^{-1}\right)$.

\subsection{ESR measurements}

In ESR measurements, sample solutions were injected into the capillary tubes incubating at $25 \pm 0 \cdot 05^{\circ} \mathrm{C}$, using a Varian E-112 EPR instrument fitted with temperature controller with the usual spectrometer settings such as $100 \mathrm{kHz}$ modulation amplitude, microwave power $8 \mathrm{~mW}$, scan range $100 \mathrm{G}$ and scan speed $8 \mathrm{~min}$.

\section{Results and discussion}

The measured and fitted SANS distribution for $\mathrm{NaC}$ and $\mathrm{NaDC}$ secondary micelles in $0 \cdot 1 \mathrm{M}$ solutions are shown in figure 1 . Best-fit results are found for monodisperse ellipsoidal models of $\mathrm{NaC}$ and $\mathrm{NaDC}$ micelles. The SANS distributions developed correlation peaks around $Q=0 \cdot 12$ and $0 \cdot 1 \AA^{-1}$ for $\mathrm{NaC}$ and $\mathrm{NaDC}$ micelles. The shifting to shorter $Q$ values for constant concentrations of bile salt indicate increasing average intermicellar distance $\left(d=2 \pi / Q_{\max }\right)$ with $Q_{\max }=Q$ at peak position, i.e. when secondary micelles are formed due to the association of primary micelles, the number density of primary micelles tends to decrease. This effect causes an increase in the average intermicellar distance. The other micellar parameters obtained from SANS data after applying Hayter-Penfold type of analysis for $\mathrm{NaC}$ and $\mathrm{NaDC}$ are presented in table 1 . The ellipsoidal micelles of pure $\mathrm{NaC}$ and $\mathrm{NaDC}$ with semi-minor axes $(a=c=8 \AA)$ and semi-major axis ( $b=18.4 \AA$ and $31.7 \AA$ ) produce best-fit results and correspond to the 


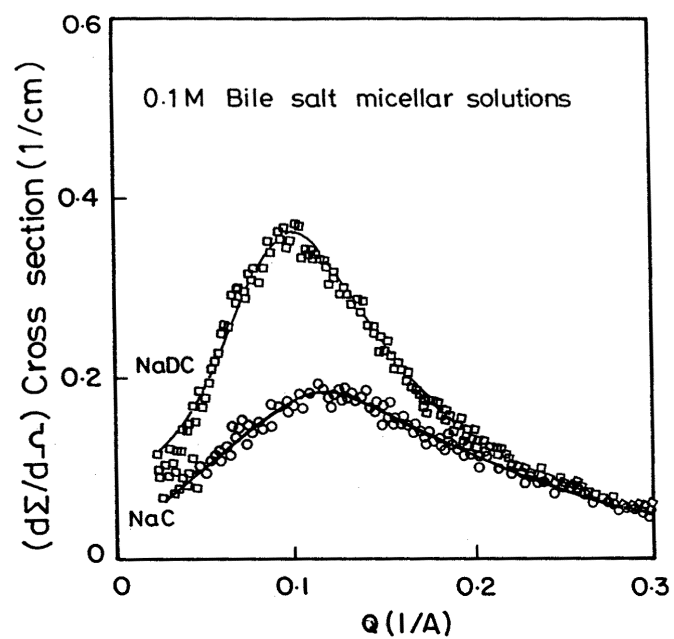

Figure 1. Small angle neutron scattering data of sodium cholate $(\mathrm{NaC})$ and sodium deoxycholate $(\mathrm{NaDC})$ secondary micelles in $\mathrm{D}_{2} \mathrm{O}$ solution at $25^{\circ} \mathrm{C}$.

Table 1. Micellar parameters of $0 \cdot 1 \mathrm{M} \mathrm{D}_{2} \mathrm{O}$ solutions of sodium cholate and sodium deoxycholate secondary micelles at $298 \mathrm{~K}$.

\begin{tabular}{lcc}
\hline Parameters & $\mathrm{NaC}$ & $\mathrm{NaDC}$ \\
\hline$R(\AA)$ & $10 \cdot 6$ & $12 \cdot 7$ \\
$a=c(\AA)$ & $8 \cdot 0$ & $8 \cdot 0$ \\
$b(\AA)$ & $18 \cdot 4$ & $31 \cdot 7$ \\
$N_{B S}$ & $9 \cdot 1$ & $16 \cdot 2$ \\
{$[\mathrm{mic}] \times 10^{3}\left(\mathrm{~mol} \mathrm{dm}^{-3}\right)$} & $9 \cdot 34$ & $5 \cdot 86$ \\
$N_{m} \times 10^{-18}\left(\mathrm{dm}^{-3}\right)$ & $5 \cdot 62$ & $3 \cdot 53$ \\
$d(\AA)$ & $52 \cdot 4$ & $62 \cdot 8$ \\
$\alpha$ & $0 \cdot 79$ & $0 \cdot 62$ \\
$\kappa^{-1}(\AA)$ & $4 \cdot 1$ & $3 \cdot 5$ \\
$U(r) / U(T) \times 10^{-5}\left(K_{B} T\right)$ units & $0 \cdot 0115$ & $0 \cdot 0227$ \\
$\tau_{B} \times 10^{9}(\mathrm{~s})$ & $3 \cdot 2$ & $5 \cdot 8$ \\
$\tau_{C} \times 10^{9}(\mathrm{~s})$ & $2 \cdot 45$ & $3 \cdot 3$ \\
$\tau_{R} \times 10^{9}(\mathrm{~s})$ & $2 \cdot 8$ & $4 \cdot 38$ \\
$R_{h}(\AA)$ & $14 \cdot 2$ & $16 \cdot 3$ \\
$R^{*}(\AA)$ & $15 \cdot 0$ & $17 \cdot 0$ \\
\hline
\end{tabular}

*Value from Ref. [18] for $\mathrm{NaDC}$; from Ref. [36] for $\mathrm{NaC}$

dimensions of secondary micelles. The equivalent spherical radii values seem to be in agreement with those in the literature ${ }^{28}$. Equation (1) representing Hayter-Penfold bestfits produces $N_{m}$ values of strongly interacting micelles in aqueous media. Bile salt micelles being anionic with $(1-\alpha)$ being the fraction of the bound counterions $\left(\mathrm{Na}^{+}\right)$per micelle, the Debye screening length $\kappa^{-1}$ is given as $\left[8 \pi N_{\mathrm{av}} e^{2} I / 1000 \xi K_{B} T\right]^{1 / 2}$ where $I$ is the ionic strength $=\left(\mathrm{CMC}+\alpha C_{s}\right) / 2$ where $C_{s}$ is the concentration of the bile salt, $\xi$ is the dielectric constant of $\mathrm{D}_{2} \mathrm{O}=78.25, K_{B}$ is the Boltzmann constant and $e$ is the electronic charge. The intermicellar interaction potential in terms of the screened coulomb 
potential of ionic micelles $U(r)$ and the contact potential $U(T)$ is taken as $U(r) / U(T)=\exp [-(r-\sigma) / \kappa r]$, where $r$ is the intermicellar distance that is equivalent to $d$ since $d \gg R$ and $\sigma=2 R$ respectively.

In figure 2, the ESR spectra of the spin probe CSL solubilised $\mathrm{NaC}$ and $\mathrm{NaDC}$ aqueous solutions are given. Since the ESR spectra recorded on blank solutions of bile salt did not give any signal and the ESR spectra of the ethanolic solution of the spin probe produced $\tau_{R}$ values very much lower than that of the micellar solutions, the observed ESR spectrum (figure 2) distinctly represents the interaction of the spin probe with the micelles ${ }^{29-31}$. Using (2)-(4), the rotational correlation times $\tau_{R}$ are calculated and listed in table 1.

$$
\tau_{R}=\left(\tau_{B} \cdot \tau_{C}\right)^{1 / 2}
$$

where $\tau_{B}=C_{1} \cdot B$ and $\tau_{C}=C_{2} \cdot C$, and

$$
\begin{aligned}
& B=-\frac{1}{2} \Delta H_{0}\left\{\left(I_{0} / I_{1}\right)^{1 / 2}-\left(I_{0} / I_{-1}\right)^{1 / 2}\right\} ; C_{1}=1.27 \times 10^{-9}, \\
& C=\frac{1}{2} \Delta H_{0}\left\{\left(I_{0} / I_{1}\right)^{1 / 2}+\left(I_{0} / I_{-1}\right)^{1 / 2}-2\right\} ; C_{1}=1.19 \times 10^{-9} .
\end{aligned}
$$

For the nitroxide spin probe used here, the principal $g$ factors and hyperfine splitting factors are: $g_{x x}=2.009 ; g_{y y}=2.006 ; g_{z z}=2.002, A_{x x}=A_{y y}=6 G ; A_{z z}=32 G . \Delta H_{0}$ refers to the peak-to-peak line width of the centre line; $I_{0}, I_{1}$ and $I_{-1}$ are the amplitudes of the low, centre and high field lines in the 3-line spectra of the spin probe. The hydrodynamic equivalent spherical radii of the micelles are calculated from the $\tau_{R}$ values using the Stokes-Einstein relation for rotational diffusion motion of the micelles, i.e., $\tau_{R}=4 \pi \eta R_{h}{ }^{3} / 3 K_{B} T$, where $\eta$ is the solvent viscosity, $T=298 \mathrm{~K}$ and $K_{B}=$ Boltzmann constant.

Regarding the secondary micellization process, bile salts differ from those of classical surfactants. This is because bile salt molecules have the hydrophobic surface at the convex side ( $\beta$ side) of the steroid nucleus and the hydrophilic surface consisting of hydroxyl and carboxylate groups at the concave side ( $\alpha$ side) of the steroid nucleus.

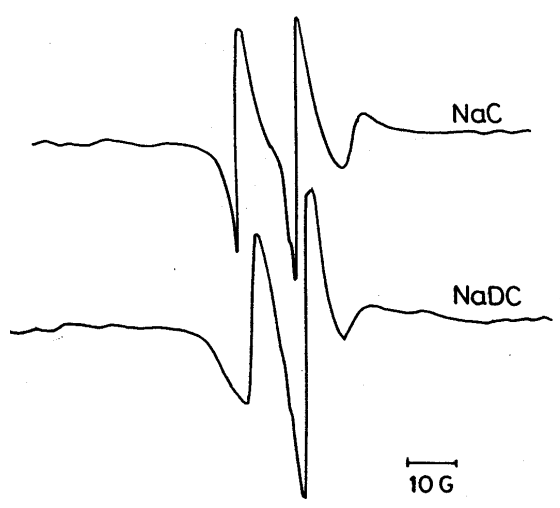

Figure 2. ESR of $0 \cdot 1 \mathrm{M}$ bile salts solubilised with the spin probe CSL. 


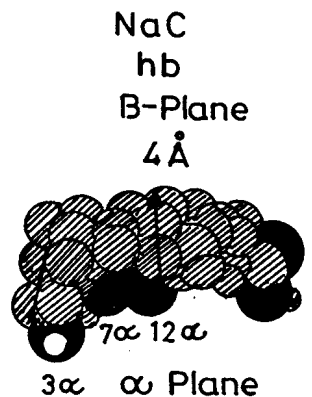

$\mathrm{NaC}$

dimer
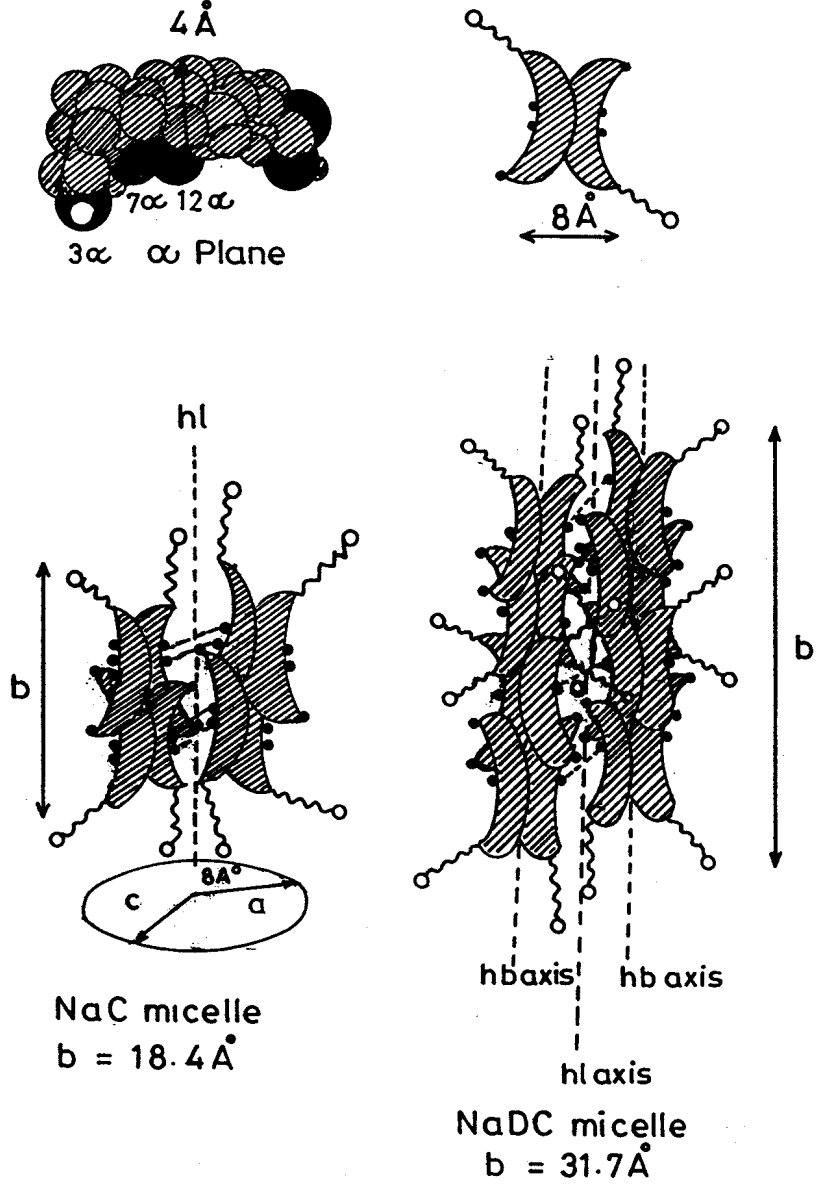

$h l=$ hydrophilic

$h b=$ hydrophobic

Figure 3. Structures of secondary micelles of sodium cholate $(\mathrm{NaC})$ and sodium deoxycholate $(\mathrm{NaDC})$.

Interaction among the hydrophobic faces results in micelle formation ${ }^{32}$. Secondary micelles are developed due to the aggregation of primary micelles favoured by the $\mathrm{H}$ bonding forces among the hydrophilic faces. During the formation of secondary micelles, rod-like micelles constituting discrete regions of both hydrophilic (H-bonding) and hydrophobic bonding exist. In figure 3, schematic representation of the super grown $\mathrm{NaC}$ and $\mathrm{NaDC}$ micelles are depicted with different hydrophobic and hydrophilic regions. Commonly the free bile acid molecules are about 20-21 $\AA$ long, from end to end. The steroid nucleus is not flat but circular in cross-section. The width of the steroid nuclei between the $\alpha$ and $\beta$ surfaces is nearly 3.5-4 $\AA$ units. Using the effective radius $(R)$ value, volume per micelle may be determined ${ }^{33}$. Knowing volume per bile salt molecule in the anhydrous sodium salt condition is $539 \AA^{3}$ and $526 \AA^{3}$ respectively for $\mathrm{NaC}$ and 
NaDC molecules, approximate $N_{B S}$ values are deduced ${ }^{34}$. Since SANS estimations involve only the hard core radii values and not the hydrodynamic ones, the anhydrous volumes of bile salt molecules are considered. In doing so, $N_{B S}$ values per super grown micelles are nearly 9.0 and 16.0 for $\mathrm{NaC}$ and $\mathrm{NaDC}$ systems. This is one reason for the higher intensity of the SANS curve for the $\mathrm{NaDC}$ than for the $\mathrm{NaC}$ micelle systems (figure 1).

Regarding the structural properties of the secondary micelles, microstructures of the $\mathrm{NaC}$ micelles as depicted in the scheme coincide well with those reported in the literature ${ }^{35}$. However bile salt secondary micelles consisting of units of tetramers $(b$ in scheme) can be built up along the horizontal way (axis $a$ ) through H-bondings or along the vertical way (axis $b$ ) which is mainly favoured by hydrophobic forces. SANS data presently predict hydrophobically grown micelles of $\mathrm{NaDC}$ rather than the other form which is based mainly on the semi-major axis value of the ellipsoid and is nearly twice as that of the $\mathrm{NaC}$ when the semi-minor axes are the same. This type of the process of growth in the micelle is favoured under high concentrations of bile salts while growth is favoured mainly by the hydrophilic forces under high ionic strengths of the medium with high concentrations of the counterions ${ }^{36}$. Counterion condensation favours micellar growth. It is observed that $\alpha$ of $\mathrm{NaDC}$ is lower than that of $\mathrm{NaC}$. This indicate clearly that screening of coulombic potentials between the micelles occurs to a greater extent in $\mathrm{NaDC}$ than in $\mathrm{NaC}$. Screened coulombic forces favour hydrophobic binding. Hence rodlike micelles with hydrophilic bonds at the central axis of the rod, seems the best-fit picture of the secondary micelle of NaDC.

Considering $R$ and $R_{h}$ values from SANS and ESR measurements, $R_{h}$ values are found to be greater. Values of $R$ are deduced from the core radii values. The discrepancy in the value is equivalent to the Debye screening length of the ionic micelles. Thus the equivalent spherical radius value evaluated from $a$ and $b$ values when corrected with hydration layer thickness, gives comparable $R_{h}$ values, i.e., the hydrodynamic semi-minor $\left(a_{h}\right)$ and semi-major $\left(b_{h}\right)$ radii values of $\mathrm{NaC}$ are $\left(a+\kappa^{-1}\right)=12 \cdot 1 \AA$ and $\left(b+\kappa^{-1}\right)=$ $22.5 \AA$. The hydrodynamic equivalent spherical radius value calculated from $a_{h}$ and $b_{h}$ $\left[=\left(a_{h}^{2} \cdot b_{h}\right)^{1 / 3}\right]$ is $14.9 \AA$. Similarly for the NaDC system the hydrodynamic equivalent spherical radius value is $16 \cdot 7 \AA$.

These values are in agreement with the $R_{h}$ values obtained from ESR measurements (table 1). The intermicellar interaction potential, $U(r) / U(T)$ in terms of $K_{B} T$ units is calculated from the SANS data (table 1) and for NaDC micelles, the values are twice as much as those for $\mathrm{NaC}$ micelles. Comparing reports on the size and shape of bile salt micelles grown as single crystals, the views may be extended as below. The hydrophobic axis parallel to the hydrophilic central axis, concentric to the hydrophilic central axis of the prolate ellipsoids of bile salt micelles $(\mathrm{NaDC})$, helically winds around the central axis if further growth along the major axis takes place. If concentration of bile salt is not very high, then short prolate micelles as in the present case would exist in solution wherein the helical nature of the hydrophobic axis may not be seen. In such a case, the observed data agree well with those of the literature ${ }^{9}$.

\section{Acknowledgement}

The authors thank the Inter-University Consortium for DAE Facilities, Government of India for financial assistance and for the permission to procure SANS data from the Dhruva Reactor at BARC, Mumbai (Project No. CRS-M-62). 


\section{References}

1. Esumi K and Ueno M (eds) 1997 Structure-performance relationships in surfactants (Surfactant Science Series) (New York: Marcel Dekker) vol 70, chap. 3, pp 147-195

2. Small D M 1971 The bile acid (New York: Plenum) vol 1, p. 302

3. Borgtstrom B, Barrowman J A and Lindstrom M 1985 In Sterols and bile acid (eds) H Danielsson and J Sjoval (Amsterdam: Elsevier)

4. Small D M, Penkett S and Chapman D 1969 Biochim. Biophys. Acta 176178

5. Bourges M, Small D M and Dervichian D G 1967 Biochim. Biophys. Acta 144189

6. Small D M 1968 J. Am. Oil Chem. Soc. 45198

7. Oakenful D G and Fisher L R 1977 J. Phys. Chem. 811838

8. Zakrzewska J, Markovic V, Vucelic D, Feigin L, Dembo A and Mogilevsky L 1990 J. Phys. Chem. 945079

9. Esposito G, Giglio E, Pavel N V and Zanobi A 1987 J. Phys. Chem. 91356

10. Hao L, Lu R, Least D G and Poulin P R 1997 J. Solution Chem. 26113

11. Coello A, Meijide F, Rodriguez Nunez E and Vazquez Tato J 1993 J. Phys. Chem. 9710186

12. Pedersen J S, Egelhaaf S and Schurtenberger P 1995 J. Phys. Chem. 991299

13. Cohen D E, Thurston G M, Chamberlin R A, Benedek G B and Carey M C 1998 Biochemistry 3714798

14. Conte G, Di Blasi R, Giglio E, Parretta A and Pavel N V 1984 J. Phys. Chem. 885720

15. O'Connor C J, Ch'ng B T and Wallace R G 1983 J. Coll. Int. Sci. 95410

16. Coello A, Meijide F, Rodriguez Nunez E and Vazquez Tato J 1996 J. Pharm. Sci. 859

17. Hjelm R P, Schteingart C D, Hofmann A F and Thiagarajan P 2000 J. Phys. Chem. B104 197

18. Lopez F, Samseth J, Mortensen K, Rosenqvist E and Rouch J 1996 Langmuir 126188

19. Igimi H and Carey M C 1980 J. Lipid Res. 2172

20. Goyal P S, Chakravarthy R, Dasannacharya B A, Desa J A E, Kelkar V K, Manohar C, Narasimhan S L, Rao K R and Valaulikar B S 1989 Physica 156471

21. Goyal P S, Dasannacharya B A, Kelkar V K, Manohar C, Srinivasa Rao K and Valaulikar B S 1991 Physica 174196

22. Kawamura H, Murata Y, Yamaguchi T, Igimi H, Tanaka M, Sugihara G and Kratohvil J P 1989 J. Phys. Chem. 933321

23. Kratohvil J P 1984 Hepatology $485 \mathrm{~S}$

24. Cannon B, Polnaszek C F, Butler K W, Erikson K E G and Smith I C P 1975 Arch. Biochim. Biophys. 167505

25. Goyal P S, Aswal V K and Joshi V 1995 In User's manual for position sensitive detector based small angle neutron scattering spectrometer, Bhabha Atomic Research Centre, Mumbai, Government of India

26. Berr S S 1987 J. Phys. Chem. 914760

27. Santhanalakshmi J, Goyal P S, Aswal V K and Vijayalakshmi G 1999 Proc. Indian Acad. Sci. (Chem. Sci.) 111651

28. Young C Y, Missel P J, Mazer N A, Benedek G B and Carey M C 1978 J. Phys. Chem. 82 1375

29. Schreier S, Polnaszek C F and Smith I C P 1978 Biochim. Biophys. Acta 515375

30. Polnaszek C F 1976 In Spin labelling theory and applications (ed.) L J Berliner (New York: Academic Press)

31. Kooser R G, Volland W V and Freed J H 1969 J. Chem. Phys. 505243

32. Blow D M and Rich A 1959 Nature (London) 1823566

33. Esposito G and Giglio E 1987 J. Phys. Chem. 91356

34. Small D M 1968 Adv. Chem. Ser. 8431

35. Dembo A T, Sosfenov N I and Feigin L A 1966 Sov. Phys. Crystallogr. (Engl. Transl.) 58119

36. Mazer N A, Carey M C, Kwasnick R F and Benedek G B 1979 Biochemistry 183064 